\title{
A kind of Bernoulli-type quasi-interpolation operator with univariate multiquadrics
}

\author{
REN-HONG WANG and MIN XU \\ Institute of Mathematical Sciences, Dalian University of Technology, \\ Dalian 116024, P.R.China \\ E-mail: xumin80@gmail.com
}

\begin{abstract}
In this paper, a kind of Bernoulli-type operator is proposed by combining a univariate multiquadric quasi-interpolation operator with the generalized Taylor polynomial. With an assumption on the shape-preserving parameter $c$, the convergence rate of the new operator is derived, which indicates that it could produce the desired precision. Numerical comparisons show that this method offers a higher degree of accuracy. Moreover, the associated algorithm is easily implemented.
\end{abstract}

Mathematical subject classification: 41A05, 41A35.

Key words: radial basis functions, multiquadrics, quasi-interpolation, Bernoulli polynomials, convergence rate.

\section{Introduction}

The standard formula for interpolating a function $f:[a, b] \rightarrow \mathbb{R}$ on scattered points and data $\left\{\left(x_{j}, f_{j}\right)\right\}_{j=0}^{N}$, where

$$
a=x_{0}<x_{1}<\cdots<x_{N}=b,
$$

has the form

$$
\mathcal{L} f(x)=\sum_{j=0}^{N} \lambda_{j} \varphi\left(x-x_{j}\right)=\sum_{j=0}^{N} \lambda_{j} \varphi_{j}(x),
$$

\#CAM-62/09. Received: 23/II/09. Accepted: 09/III/09. 
such that

$$
f\left(x_{j}\right)=\mathcal{L} f\left(x_{j}\right), \quad \text { for } 0 \leqslant j \leqslant N,
$$

where $\varphi(\cdot)$ is an interpolation kernel. Many researchers use radial basis functions (RBFs) to solve the interpolation problem (1.2)-(1.3). In particular, the multiquadrics (MQs) introduced by Hardy [10],

$$
\varphi_{j}(x):=\varphi\left(x-x_{j}\right)=\sqrt{\left(x-x_{j}\right)^{2}+c^{2}}, \quad j=0,1, \cdots, N,
$$

are of special interest, because of their spectral convergence property, see $[5,6]$. Throughout the rest of this paper, we use the notations $\varphi_{j}(\cdot)$ and $c$ to denote the MQs and their shape-preserving parameter as in (1.4), respectively. A review by Franke [9] showed that the MQ interpolation is considered as one of the best methods among 29 scattered points interpolation schemes based on accuracy, stability, efficiency, memory requirement and easy implementation. Although the MQ interpolation is always solvable when the $x_{j}$ 's are distinct [11], the associated coefficient matrix in (1.2) quickly becomes ill-conditioned as the number of points increases. There are different ways to overcome this problem. In this paper, we will focus on the quasi-interpolation method. A weaker form of (1.3), known as quasi-interpolation, holds only for polynomials of degree $\leqslant m$, i.e.,

$$
p\left(x_{j}\right)=\mathcal{L} p\left(x_{j}\right), \quad \forall p \in \Pi_{m},
$$

for all $0 \leqslant j \leqslant N$, where $\Pi_{m}$ denotes the set of polynomials of degree $\leqslant m$. Many researchers have investigated the MQ quasi-interpolation method, see $[1,2,14,15]$ for details.

Based on the idea in [7], we combine the multiquadric quasi-interpolation operator $\mathcal{L}_{\mathcal{B}}$ proposed in [3] with the generalized Taylor polynomial proposed in [8] to get a Bernoulli-type quasi-interpolation operator. The new operator could reproduce polynomials of higher degree than the operator $\mathcal{L}_{\mathcal{B}}$. We derive the convergence rate of the operator with a suitable assumption on the shapepreserving parameter $c$, and find that our operator could achieve a convergence rate of higher order by using a smaller parameter, which makes the associated quasi-interpolant less smooth. So we could use an optimal value of $c$ according to the desired smoothness and precision of the quasi-interpolant. 
The remainder of this paper is organized as follows. In Section 2, we introduce some previous results about the generalized Taylor polynomial, which plays an important role in the construction of our operator. In Section 3, we propose the Bernoulli-type quasi-interpolation operator, and study its convergence rate. In Section 4, we give numerical experiments to show that the operator is capable of producing high accuracy. In Section 5, we give the conclusions and future work.

\section{The generalized Taylor polynomial}

The generalized Taylor polynomial is an expansion in Bernoulli polynomials $B_{n}(x)$, i.e., the polynomials defined by the following generating function

$$
\frac{t e^{t x}}{e^{t}-1}=\sum_{n=0}^{\infty} B_{n}(x) \frac{t^{n}}{n !} .
$$

For any function $f$ in the class $C^{m}[a, b](a<b)$, this expansion is realized by the equation

$$
f(x)=P_{m}[f ; a, b](x)+R_{m}[f ; a, b](x), \quad x \in[a, b],
$$

where the polynomial approximation term $P_{m}[f ; a, b](x)$ is given by

$$
\begin{gathered}
P_{m}[f ; a, b](x)= \\
f(a)+\sum_{k=1}^{m} \frac{B_{k}\left(\frac{x-a}{h}\right)-B_{k}(0)}{k !} h^{k-1}\left(f^{(k-1)}(b)-f^{(k-1)}(a)\right)
\end{gathered}
$$

and the remainder term $R_{m}[f ; a, b](x)$ is given by

$$
\begin{gathered}
R_{m}[f ; a, b](x)= \\
\frac{h^{m-1}}{m !} \int_{a}^{b} f^{(m)}(t)\left(B_{m}\left(\frac{b-t}{h}\right)-B_{m}\left(\frac{x-t}{h}-\left[\frac{x-t}{h}\right]\right)\right) \mathrm{dt},
\end{gathered}
$$

with $h=b-a$. The polynomial approximant $P_{m}[f ; a, b](x)$ has the following properties:

$$
\begin{aligned}
& \lim _{b \rightarrow a} P_{m}[f ; a, b](x)=T_{m}[f ; a](x), \\
& P_{m}[f ; a, b](a)=f(a), \quad P_{m}[f ; a, b](b)=f(b),
\end{aligned}
$$


where $T_{m}[f ; a](x)$ is the $m$ th Taylor polynomial of $f$ about the point $a$. Due to the property (2.5), we call $P_{m}[f ; a, b](x)$ the generalized Taylor polynomial. The following two theorems give bounds for the remainder term $R_{m}[f ; a, b](x)$, see [7].

Theorem 2.1. Let $f \in C^{m}[c, d]$ and $x \in[c, d]$. For the remainder term, we have

$$
\left|R_{m}[f ; a, b](x)\right| \leqslant \begin{cases}C(m)\left\|f^{(m)}\right\|_{\infty}(b-x)^{m}, & c \leqslant x \leqslant a, \\ C(m)\left\|f^{(m)}\right\|_{\infty}(b-a)^{m}, & a \leqslant x \leqslant b, \\ C(m)\left\|f^{(m)}\right\|_{\infty}(x-a)^{m}, & b \leqslant x \leqslant d,\end{cases}
$$

where $\|\cdot\|_{\infty}$ denotes the sup-norm on $[c, d]$ and

$$
C(m)=\frac{1}{m !}\left(1+\sum_{k=1}^{m} \sum_{j=1}^{k}\left(\begin{array}{l}
m \\
k
\end{array}\right)\left(\begin{array}{l}
k \\
j
\end{array}\right)\left|B_{k-j}(0)\right|\right), \quad m=1,2, \cdots .
$$

Theorem 2.2. Let $f \in C^{m+1}[c, d]$ and $x \in[c, d]$. For the remainder term, we have

$$
\left|R_{m}[f ; a, b](x)\right| \leqslant \begin{cases}C(m+1)\left\|f^{(m+1)}\right\|_{\infty}(b-x)^{m+1}, & c \leqslant x \leqslant a, \\ C(m+1)\left\|f^{(m+1)}\right\|_{\infty}(b-a)^{m+1}, & a \leqslant x \leqslant b, \\ C(m+1)\left\|f^{(m+1)}\right\|_{\infty}(x-a)^{m+1}, & b \leqslant x \leqslant d,\end{cases}
$$

where $C(m)$ is defined by (2.8).

\section{The Bernoulli-type quasi-interpolation operator}

The quasi-interpolation operator $\mathcal{L}_{\mathcal{B}}$ is defined as follows:

$$
\left(\mathcal{L}_{\mathcal{B}} f\right)(x)=f\left(x_{0}\right) \psi_{0}(x)+\sum_{i=1}^{N-1} f\left(x_{i}\right) \psi_{i}(x)+f\left(x_{N}\right) \psi_{N}(x), \quad x \in[a, b],
$$

where

$$
\begin{aligned}
\psi_{0}(x) & =\frac{1}{2} c^{2} \int_{-\infty}^{x_{0}} \frac{1}{\left[(x-\theta)^{2}+c^{2}\right]^{3 / 2}} \mathrm{~d} \theta \\
& +\frac{1}{2} c^{2} \int_{x_{0}}^{x_{1}} \frac{\left(x_{1}-\theta\right) /\left(x_{1}-x_{0}\right)}{\left[(x-\theta)^{2}+c^{2}\right]^{3 / 2}} \mathrm{~d} \theta \\
& =\frac{1}{2}+\frac{\varphi_{1}(x)-\varphi_{0}(x)}{2\left(x_{1}-x_{0}\right)}
\end{aligned}
$$




$$
\begin{aligned}
\psi_{N}(x) & =\frac{1}{2} c^{2} \int_{x_{N}}^{\infty} \frac{1}{\left[(x-\theta)^{2}+c^{2}\right]^{3 / 2}} \mathrm{~d} \theta \\
& +\frac{1}{2} c^{2} \int_{x_{N-1}}^{x_{N}} \frac{\left(\theta-x_{N-1}\right) /\left(x_{N}-x_{N-1}\right)}{\left[(x-\theta)^{2}+c^{2}\right]^{3 / 2}} \mathrm{~d} \theta \\
& =\frac{1}{2}-\frac{\varphi_{N}(x)-\varphi_{N-1}(x)}{2\left(x_{N}-x_{N-1}\right)},
\end{aligned}
$$

and

$$
\begin{aligned}
\psi_{i}(x) & =\frac{1}{2} c^{2} \int_{x_{i-1}}^{x_{i+1}} \frac{B_{i}(\theta)}{\left[(x-\theta)^{2}+c^{2}\right]^{3 / 2}} \mathrm{~d} \theta \\
& =\frac{\varphi_{i+1}(x)-\varphi_{i}(x)}{2\left(x_{i+1}-x_{i}\right)}-\frac{\varphi_{i}(x)-\varphi_{i-1}(x)}{2\left(x_{i}-x_{i-1}\right)}
\end{aligned}
$$

for $i=1,2, \cdots, N-1$, where $B_{i}(\theta)$ is the piecewise linear hat function having the knots $\left\{x_{i-1}, x_{i}, x_{i+1}\right\}$, and satisfying $B_{i}\left(x_{i}\right)=1$. The operator $\mathcal{L}_{\mathcal{B}}$ reproduces polynomials of zero degree, i.e.,

$$
\psi_{0}(x)+\sum_{i=1}^{N-1} \psi_{i}(x)+\psi_{N}(x)=1 .
$$

By combining the operator $\mathcal{L}_{\mathcal{B}}$ with the $m$ th generalized Taylor polynomial, we propose a kind of Bernoulli-type quasi-interpolation operator $\mathcal{L}_{B_{m}}$ as follows

$$
\left(\mathcal{L}_{B_{m}} f\right)(x)=\sum_{i=0}^{N} \psi_{i}(x) P_{m}\left[f ; x_{i}, x_{i+1}\right](x)
$$

with $x_{N+1}=x_{N-1}$. The operator $\mathcal{L}_{B_{m}}$ has the following polynomial reproduction property.

Theorem 3.1. The Bernoulli-type operator $\mathcal{L}_{B_{m}}$ reproduces all polynomials of degree $\leqslant m$, i.e.,

$$
\mathcal{L}_{B_{m}} f=f, \quad \forall f \in \Pi_{m} .
$$

Proof. It is easy to show that

$$
\int_{x_{i}}^{x_{i+1}}\left(B_{m}\left(\frac{x_{i+1}-t}{h_{i}}\right)-B_{m}\left(\frac{x-t}{h_{i}}-\left[\frac{x-t}{h_{i}}\right]\right)\right) \mathrm{dt}=0,
$$


where $h_{i}=x_{i+1}-x_{i}$. Then, by (2.2) and (2.4) we get

$$
P_{m}\left[f ; x_{i}, x_{i+1}\right](x)=f(x), \quad \forall f \in \Pi_{m} .
$$

Combining it with (3.5), we have (3.7).

Remark 3.1. If $P_{m}\left[f ; x_{i}, x_{i+1}\right](x)$ in (3.6) is replaced by the mth Taylor polynomials $T_{m}\left[f ; x_{i}\right]$, the corresponding operator also reproduces polynomials of degree $\leqslant m$. In this case, however, we need to compute the derivatives of $f$ up to order $m$, which is one greater than the order of derivatives needed in $\mathcal{L}_{B_{m}}$.

In order to study the convergence rates of the new operator, we introduce the following notations

$$
\begin{aligned}
& I_{\rho}(x)=[x-\rho, x+\rho], \quad \rho>0, \\
& r=\frac{1}{2} \max \left\{\left|x_{1}-x_{0}\right|,\left|x_{2}-x_{1}\right|, \cdots,\left|x_{N}-x_{N-1}\right|\right\}, \\
& M=\max _{x \in[a, b]} \#\left(I_{r}(x) \cap X\right),
\end{aligned}
$$

where $X=\left\{x_{0}, x_{1}, \cdots, x_{N}\right\}$ and $\#(\cdot)$ denotes the cardinality function. Therefore, $M$ is the maximum number of points from $X$ contained in an interval $I_{r}(x)$. For the convergence rate of the Bernoulli-type quasi-interpolation operator $\mathcal{L}_{B_{m}}$, we have the following theorem.

Theorem 3.2. Assume that c satisfies

$$
c \leqslant D r^{l}
$$

where $D$ is a positive constant, and $l$ is a positive integer. If $f \in C^{m}[a, b]$, then

$$
\left\|\mathcal{L}_{B_{m}} f-f\right\|_{\infty} \leqslant K M\left\|f^{(m)}\right\|_{\infty} \mathcal{E}_{l, m}(r),
$$

where

$$
\mathcal{E}_{l, m}(r)= \begin{cases}r|\ln r|, & m=1, l=1, \\ r, & m=1, l>1, \\ r^{m}, & m>1, m \leqslant 2 l-1, \\ r^{2 l-1}, & m>1, m>2 l-1\end{cases}
$$

and $K$ is a positive constant independent of $x$ and $X$. 
Proof. For each pair $x_{i}, x_{i+1}$, we set

$$
d\left[x_{i}, x_{i+1}\right](x)= \begin{cases}x_{i+1}-x, & x \leqslant x_{i}, \\ x_{i+1}-x_{i}, & x_{i} \leqslant x \leqslant x_{i+1}, \\ x-x_{i}, & x_{i+1} \leqslant x\end{cases}
$$

and

$$
d^{m}\left[x_{i}, x_{i+1}\right](x)=\left|d\left[x_{i}, x_{i+1}\right](x)\right|^{m} .
$$

We have

$$
\begin{aligned}
\left|\left(\mathcal{L}_{B_{m}} f\right)(x)-f(x)\right| & =\left|\sum_{i=0}^{N} \psi_{i}(x)\left(P_{m}\left[f ; x_{i}, x_{i+1}\right](x)-f(x)\right)\right| \\
& \leqslant \sum_{i=0}^{N} \psi_{i}(x)\left|P_{m}\left[f ; x_{i}, x_{i+1}\right](x)-f(x)\right| \\
& \leqslant C(m)\left\|f^{(m)}\right\|_{\infty} S_{l, m}(x),
\end{aligned}
$$

where $S_{l, m}(x)=\sum_{i=0}^{N} \psi_{i}(x) d^{m}\left[x_{i}, x_{i+1}\right](x)$.

Let

$$
\begin{gathered}
n=\left[\frac{b-a}{2 r}\right]+1, \\
Q_{\rho}(u)=(u-\rho, u+\rho], \quad u \in[a, b], \quad \rho>0,
\end{gathered}
$$

and

$$
T_{j}=Q_{r}(x-2 r j) \cup Q_{r}(x+2 r j), \quad j=0,1, \cdots, n,
$$

where [.] denotes the integer part of the argument. Obviously, the set $\cup_{j=-n}^{n} Q_{r}(x+2 r j)$ is a covering of $[a, b]$ with half open intervals. Therefore, for each $i \in\{0,1, \cdots, N\}$ there exists a unique $j \in\{0,1, \cdots, n\}$ such that $x_{i} \in T_{j}$. When $j \geqslant 2$, the following inequalities hold:

$$
\begin{gathered}
(2 j-1) r \leqslant\left|x-x_{i}\right| \leqslant(2 j+1) r, \\
(2(j-1)-1) r \leqslant\left|x-\xi_{i}\right| \leqslant(2(j+1)+1) r, \text { for } \xi_{i} \in\left[x_{i-1}, x_{i+1}\right],
\end{gathered}
$$

and

$$
\left|d\left[x_{i}, x_{i+1}\right](x)\right| \leqslant(2(j+1)+1) r .
$$




\begin{tabular}{|c|c|c|c|c|c|c|c|c|}
\hline \multicolumn{3}{|c|}{$S_{B_{m}}\left(f_{1}\right)$} & \multicolumn{3}{|c|}{$\mathcal{L}_{B_{m}}\left(f_{1}\right)$} & \multicolumn{3}{|c|}{$\mathcal{L}_{\mathcal{B}}\left(f_{1}\right)$} \\
\hline$(\mu, m)$ & $\varepsilon_{\text {mean }}$ & $\varepsilon_{\max }$ & $(l, m)$ & $\varepsilon_{\text {mean }}$ & $\varepsilon_{\max }$ & $l$ & $\varepsilon_{\text {mean }}$ & $\varepsilon_{\max }$ \\
\hline$(2,1)$ & 0.001050 & 0.004954 & $(2,1)$ & 0.000398 & 0.001875 & \multirow{3}{*}{2} & \multirow{3}{*}{0.001016} & \multirow{3}{*}{0.004523} \\
\hline$(2,2)$ & 0.001062 & 0.004715 & $(2,2)$ & 0.000374 & 0.001639 & & & \\
\hline$(2,3)$ & 0.001490 & 0.005153 & $(2,3)$ & 0.000304 & 0.002264 & & & \\
\hline$(3,1)$ & 0.000476 & 0.003314 & $(3,1)$ & 0.000395 & 0.001873 & \multirow{3}{*}{3} & \multirow{3}{*}{0.001000} & \multirow{3}{*}{0.004505} \\
\hline$(3,2)$ & 0.000333 & 0.002302 & $(3,2)$ & 0.000370 & 0.001642 & & & \\
\hline$(3,3)$ & 0.000206 & 0.001096 & $(3,3)$ & 0.000296 & 0.002268 & & & \\
\hline$(4,1)$ & 0.000457 & 0.003233 & $(4,1)$ & 0.000295 & 0.001873 & \multirow{3}{*}{4} & \multirow{3}{*}{0.001000} & \multirow{3}{*}{0.004505} \\
\hline$(4,2)$ & 0.000259 & 0.001908 & $(4,2)$ & 0.000370 & 0.001642 & & & \\
\hline$(4,3)$ & 0.000136 & 0.001460 & $(4,3)$ & 0.000296 & 0.002268 & & & \\
\hline
\end{tabular}

Table 1 - Saddle.

It follows from the definition of $M$ that

$$
\begin{aligned}
& 1 \leqslant \#\left(X \cap T_{0}\right) \leqslant M, \\
& 1 \leqslant \#\left(X \cap T_{j}\right) \leqslant 2 M, \quad j=1,2, \cdots, n .
\end{aligned}
$$

When $x_{0} \in T_{j}(j \geqslant 2)$, we have

$$
\begin{aligned}
\psi_{0}(x) & \leqslant \frac{1}{2} c^{2} \int_{-\infty}^{x_{0}} \frac{1}{|x-\theta|^{3}} \mathrm{~d} \theta+\frac{1}{2} c^{2} \frac{1}{\left[\left(x-\xi_{0}\right)^{2}+c^{2}\right]^{3 / 2}} \int_{x_{0}}^{x_{1}} \frac{\left(x_{1}-\theta\right)}{\left(x_{1}-x_{0}\right)} \mathrm{d} \theta \\
& \leqslant \frac{1}{4} c^{2}\left|x-x_{0}\right|^{-2}+\frac{1}{4} c^{2}\left(x_{1}-x_{0}\right)\left|x-\xi_{0}\right|^{-3} \\
& \leqslant \frac{1}{4} c^{2}\left[(2 j-1)^{-2} r^{-2}+2(2 j-3)^{-3} r^{-2}\right] \\
& \leqslant c^{2} r^{-2}(2 j-3)^{-2}
\end{aligned}
$$

where $\xi_{0} \in\left[x_{0}, x_{1}\right]$. Similarly, for $x_{N} \in T_{j}(j \geqslant 2)$, we have

$$
\psi_{N}(x) \leqslant c^{2} r^{-2}(2 j-3)^{-2} .
$$

When $x_{i}(i=1,2, \cdots, N-1)$ belong to $T_{j}(j \geqslant 2)$, we have

$$
\begin{aligned}
\psi_{i}(x) & \leqslant \frac{1}{2} c^{2} \frac{1}{\left[\left(x-\xi_{i}\right)^{2}+c^{2}\right]^{3 / 2}} \int_{x_{i-1}}^{x_{i+1}} B_{i}(\theta) \mathrm{d} \theta \\
& \leqslant \frac{1}{4} c^{2}\left(x_{i+1}-x_{i-1}\right)\left|x-\xi_{i}\right|^{-3} \\
& \leqslant c^{2} r^{-2}(2 j-3)^{-2},
\end{aligned}
$$

where $\xi_{i} \in\left[x_{i-1}, x_{i+1}\right]$. 


\begin{tabular}{|c|c|c|c|c|c|c|c|c|}
\hline \multicolumn{3}{|c|}{$S_{B_{m}}\left(f_{2}\right)$} & \multicolumn{3}{|c|}{$\mathcal{L}_{B_{m}}\left(f_{2}\right)$} & \multicolumn{3}{|c|}{$\mathcal{L}_{\mathcal{B}}\left(f_{2}\right)$} \\
\hline$(\mu, m)$ & $\varepsilon_{\text {mean }}$ & $\varepsilon_{\max }$ & $(l, m)$ & $\varepsilon_{\text {mean }}$ & $\varepsilon_{\max }$ & $l$ & $\varepsilon_{\text {mean }}$ & $\varepsilon_{\max }$ \\
\hline$(2,1)$ & 0.002145 & 0.005623 & $(2,1)$ & 0.000449 & 0.000895 & & & \\
\hline$(2,2)$ & 0.000312 & 0.000842 & $(2,2)$ & 0.000057 & 0.000246 & 2 & 0.001156 & 0.002189 \\
\hline$(2,3)$ & 0.000586 & 0.002344 & $(2,3)$ & 0.000036 & 0.000145 & & & \\
\hline$(3,1)$ & 0.000583 & 0.001620 & $(3,1)$ & 0.000445 & 0.000896 & & & \\
\hline$(3,2)$ & 0.000058 & 0.000247 & $(3,2)$ & 0.000056 & 0.000246 & 3 & 0.001143 & 0.002200 \\
\hline$(3,3)$ & 0.000079 & 0.000323 & $(3,3)$ & 0.000033 & 0.000143 & & & \\
\hline$(4,1)$ & 0.000510 & 0.001447 & $(4,1)$ & 0.000445 & 0.000896 & & & \\
\hline$(4,2)$ & 0.000039 & 0.000255 & $(4,2)$ & 0.000056 & 0.000246 & 4 & 0.001143 & 0.002200 \\
\hline$(4,3)$ & 0.000025 & 0.000113 & $(4,3)$ & 0.000033 & 0.000143 & & & \\
\hline
\end{tabular}

Table 2 - Sphere.

Then we can get

$$
\begin{aligned}
S_{l, m}(x) & \leqslant \sum_{x_{i} \in T_{0}, T_{1}} \psi_{i}(x) d^{m}\left[x_{i}, x_{i+1}\right](x)+\sum_{j=2}^{n} \sum_{x_{i} \in T_{j}} \psi_{i}(x) d^{m}\left[x_{i}, x_{i+1}\right](x) \\
& \leqslant M(3 r)^{m}+2 M(5 r)^{m}+2 M \sum_{j=2}^{n} c^{2} r^{-2}(2 j-3)^{-2}((2 j+3) r)^{m} \\
& \leqslant 2 M(5 r)^{m}+2 M(5 r)^{m}+2 M c^{2} r^{m-2} \sum_{j=2}^{n}(2 j-3)^{-2}(2 j+3)^{m} \\
& \leqslant 2 M\left((5 r)^{m}+(5 r)^{m}+c^{2} r^{m-2} \sum_{j=1}^{n} j^{-2}(5 j)^{m}\right) \\
& \left.\leqslant 2 M 5^{m}\left(2 r^{m}+c^{2} r^{m-2} \sum_{j=1}^{n} j^{m-2}\right)_{j=1}^{n} j_{j}^{m-2}\right) .
\end{aligned}
$$

Case 1: $(m=1)$.

$$
\begin{aligned}
& \text { If } l=1, \quad 2 r^{m}+D^{2} r^{2 l+m-2} \sum_{j=1}^{n} j^{m-2}=\mathcal{O}(r|\ln r|) . \\
& \text { If } l>1, \quad 2 r^{m}+D^{2} r^{2 l+m-2} \sum_{j=1}^{n} j^{m-2}=\mathcal{O}(r) .
\end{aligned}
$$




\begin{tabular}{|c|c|c|c|c|c|c|c|c|}
\hline \multicolumn{3}{|c|}{$S_{B_{m}}\left(f_{3}\right)$} & \multicolumn{3}{|c|}{$\mathcal{L}_{B_{m}}\left(f_{3}\right)$} & \multicolumn{3}{|c|}{$\mathcal{L}_{\mathcal{B}}\left(f_{3}\right)$} \\
\hline$(\mu, m)$ & $\varepsilon_{\text {mean }}$ & $\varepsilon_{\max }$ & $(l, m)$ & $\varepsilon_{\text {mean }}$ & $\varepsilon_{\max }$ & $l$ & $\varepsilon_{\text {mean }}$ & $\varepsilon_{\max }$ \\
\hline$(2,1)$ & 0.006604 & 0.038815 & $(2,1)$ & 0.001952 & 0.014873 & & & \\
\hline$(2,2)$ & 0.004710 & 0.031367 & $(2,2)$ & 0.003327 & 0.034084 & 2 & 0.006244 & 0.035059 \\
\hline$(2,3)$ & 0.013455 & 0.062821 & $(2,3)$ & 0.003365 & 0.039092 & & & \\
\hline$(3,1)$ & 0.002522 & 0.021627 & $(3,1)$ & 0.001943 & 0.014863 & & & \\
\hline$(3,2)$ & 0.002466 & 0.027527 & $(3,2)$ & 0.003300 & 0.034080 & 3 & 0.006172 & 0.034972 \\
\hline$(3,3)$ & 0.002138 & 0.016732 & $(3,3)$ & 0.003263 & 0.039076 & & & \\
\hline$(4,1)$ & 0.002405 & 0.021752 & $(4,1)$ & 0.001943 & 0.014863 & & & \\
\hline$(4,2)$ & 0.002170 & 0.034048 & $(4,2)$ & 0.003300 & 0.034080 & 4 & 0.006172 & 0.034972 \\
\hline$(4,3)$ & 0.001542 & 0.024101 & $(4,3)$ & 0.003263 & 0.039076 & & & \\
\hline
\end{tabular}

Table 3 - Cliff.

Case 2: $(m>1)$.

$$
\begin{aligned}
& \text { If } m \leqslant 2 l-1, \quad 2 r^{m}+D^{2} r^{2 l+m-2} \sum_{j=1}^{n} j^{m-2}=\mathcal{O}\left(r^{m}\right) . \\
& \text { If } m>2 l-1, \quad 2 r^{m}+D^{2} r^{2 l+m-2} \sum_{j=1}^{n} j^{m-2}=\mathcal{O}\left(r^{2 l-1}\right) .
\end{aligned}
$$

By using Theorem 2.2, we can prove the following theorem in an analogous manner.

Theorem 3.3. Assume that c satisfies

$$
c \leqslant D r^{l}
$$

where $D$ is a positive constant, and $l$ is a positive integer. If $f \in C^{m+1}[a, b]$, then

$$
\left\|\mathcal{L}_{B_{m}} f-f\right\|_{\infty} \leqslant K^{\prime} M\left\|f^{(m+1)}\right\|_{\infty} \mathcal{E}_{l, m}^{\prime}(r),
$$

where

$$
\mathcal{E}_{l, m}^{\prime}(r)= \begin{cases}r^{2 l-1}, & m+1 \geqslant 2 l-1, \\ r^{m+1}, & m+1<2 l-1\end{cases}
$$

and $K^{\prime}$ is a positive constant independent of $x$ and $X$.

Remark 3.2. From Theorem 3.3, we note that the convergence rate do not always achieve the expected maximum $m+1$. If the basis functions are replaced by $B$ splines (for instance, $\psi_{j}(x)$ degenerate into linear B-splines when $c$ is chosen to be 0$)$, the convergence rate will achieve $m+1$. However, the associated quasi-interpolant is finitely differentiable, which is less smooth than $\mathcal{L}_{B_{m}} f(x)$. 


\section{Numerical experiments}

We consider the following functions on the interval $[0,1]$

\begin{tabular}{|c|c|c|c|c|c|c|c|c|}
\hline \multicolumn{3}{|c|}{$S_{B_{m}}\left(f_{4}\right)$} & \multicolumn{3}{|c|}{$\mathcal{L}_{B_{m}}\left(f_{4}\right)$} & \multicolumn{3}{|c|}{$\mathcal{L}_{\mathcal{B}}\left(f_{4}\right)$} \\
\hline$(\mu, m)$ & $\varepsilon_{\text {mean }}$ & $\varepsilon_{\max }$ & $(l, m)$ & $\varepsilon_{\text {mean }}$ & $\varepsilon_{\max }$ & $l$ & $\varepsilon_{\text {mean }}$ & $\varepsilon_{\max }$ \\
\hline$(2,1)$ & 0.002590 & 0.007116 & $(2,1)$ & 0.000545 & 0.001645 & & & \\
\hline$(2,2)$ & 0.001897 & 0.005956 & $(2,2)$ & 0.000394 & 0.000993 & 2 & 0.001394 & 0.004020 \\
\hline$(2,3)$ & 0.001138 & 0.006015 & $(2,3)$ & 0.000185 & 0.000738 & & & \\
\hline$(3,1)$ & 0.000681 & 0.003277 & $(3,1)$ & 0.000542 & 0.001642 & & & \\
\hline$(3,2)$ & 0.000378 & 0.001727 & $(3,2)$ & 0.000388 & 0.000986 & 3 & 0.001395 & 0.003998 \\
\hline$(3,3)$ & 0.000175 & 0.000940 & $(3,3)$ & 0.000181 & 0.000736 & & & \\
\hline$(4,1)$ & 0.000618 & 0.002978 & $(4,1)$ & 0.000542 & 0.001642 & & & \\
\hline$(4,2)$ & 0.000270 & 0.001163 & $(4,2)$ & 0.000388 & 0.000986 & 4 & 0.001369 & 0.003998 \\
\hline$(4,3)$ & 0.000089 & 0.000575 & $(4,3)$ & 0.000181 & 0.000736 & & & \\
\hline
\end{tabular}

Table 4 - Gentle.

\begin{tabular}{|c|c|c|c|c|c|c|c|c|}
\hline \multicolumn{3}{|c|}{$S_{B_{m}}\left(f_{5}\right)$} & \multicolumn{3}{|c|}{$\mathcal{L}_{B_{m}}\left(f_{5}\right)$} & \multicolumn{3}{|c|}{$\mathcal{L}_{\mathcal{B}}\left(f_{5}\right)$} \\
\hline$(\mu, m)$ & $\varepsilon_{\text {mean }}$ & $\varepsilon_{\max }$ & $(l, m)$ & $\varepsilon_{\text {mean }}$ & $\varepsilon_{\max }$ & $l$ & $\varepsilon_{\text {mean }}$ & $\varepsilon_{\max }$ \\
\hline$(2,1)$ & 0.002358 & 0.012532 & $(2,1)$ & 0.001700 & 0.006483 & \multirow{3}{*}{2} & \multirow{3}{*}{0.004096} & \multirow{3}{*}{0.014145} \\
\hline$(2,2)$ & 0.002950 & 0.015868 & $(2,2)$ & 0.001929 & 0.007393 & & & \\
\hline$(2,3)$ & 0.004950 & 0.019728 & $(2,3)$ & 0.001762 & 0.009003 & & & \\
\hline$(3,1)$ & 0.001930 & 0.011016 & $(3,1)$ & 0.001692 & 0.006477 & \multirow{3}{*}{3} & \multirow{3}{*}{0.004034} & \multirow{3}{*}{0.014091} \\
\hline$(3,2)$ & 0.001501 & 0.009079 & $(3,2)$ & 0.001909 & 0.007375 & & & \\
\hline$(3,3)$ & 0.000909 & 0.005278 & $(3,3)$ & 0.001729 & 0.009025 & & & \\
\hline$(4,1)$ & 0.001945 & 0.011413 & $(4,1)$ & 0.001992 & 0.006477 & \multirow{3}{*}{4} & \multirow{3}{*}{0.004034} & \multirow{3}{*}{0.014091} \\
\hline$(4,2)$ & 0.001323 & 0.008184 & $(4,2)$ & 0.001909 & 0.007375 & & & \\
\hline$(4,3)$ & 0.000815 & 0.006381 & $(4,3)$ & 0.001729 & 0.009025 & & & \\
\hline
\end{tabular}

Table 5 - Steep.

\begin{tabular}{|c|c|c|c|c|c|c|c|c|}
\hline \multicolumn{3}{|c|}{$S_{B_{m}}\left(f_{6}\right)$} & \multicolumn{3}{|c|}{$\mathcal{L}_{B_{m}}\left(f_{6}\right)$} & \multicolumn{3}{|c|}{$\mathcal{L}_{\mathcal{B}}\left(f_{6}\right)$} \\
\hline$(\mu, m)$ & $\varepsilon_{\text {mean }}$ & $\varepsilon_{\max }$ & $(l, m)$ & $\varepsilon_{\text {mean }}$ & $\varepsilon_{\max }$ & $l$ & $\varepsilon_{\text {mean }}$ & $\varepsilon_{\max }$ \\
\hline$(2,1)$ & 0.007669 & 0.034957 & $(2,1)$ & 0.004663 & 0.015048 & & & \\
\hline$(2,2)$ & 0.005271 & 0.025436 & $(2,2)$ & 0.006369 & 0.021784 & 2 & 0.010340 & 0.035225 \\
\hline$(2,3)$ & 0.025296 & 0.067861 & $(2,3)$ & 0.007923 & 0.027253 & & & \\
\hline$(3,1)$ & 0.005122 & 0.021099 & $(3,1)$ & 0.004645 & 0.015044 & & & \\
\hline$(3,2)$ & 0.004379 & 0.024620 & $(3,2)$ & 0.006328 & 0.021752 & 3 & 0.010208 & 0.035158 \\
\hline$(3,3)$ & 0.003523 & 0.020488 & $(3,3)$ & 0.007798 & 0.027159 & & & \\
\hline$(4,1)$ & 0.005026 & 0.022762 & $(4,1)$ & 0.004645 & 0.015044 & & & \\
\hline$(4,2)$ & 0.004233 & 0.024080 & $(4,2)$ & 0.006328 & 0.021752 & 4 & 0.010208 & 0.035158 \\
\hline$(4,3)$ & 0.003020 & 0.018326 & $(4,3)$ & 0.007797 & 0.027159 & & & \\
\hline
\end{tabular}

Table 6-Exponential. 


$$
\begin{aligned}
\text { Saddle } f_{1}= & \frac{1.25}{6+6(3 x-1)^{2}}, \\
\text { Sphere } f_{2}= & \frac{\sqrt{64-81(x-0.5)^{2}}}{9}-0.5, \\
\text { Cliff } f_{3}= & \frac{\tanh (-9 x+1)}{2}+0.5, \\
\text { Gentle } f_{4}= & \frac{\exp \left(-\frac{81}{16}(x-0.5)^{2}\right)}{3}, \\
\text { Steep } \quad f_{5}= & \frac{\exp \left(-\frac{81}{4}(x-0.5)^{2}\right)}{3}, \\
\text { Exponential } \quad f_{6}= & 0.75 \exp \left(-\frac{(9 x-2)^{2}}{4}\right)+0.75 \exp \left(-\frac{(9 x+1)^{2}}{49}\right) \\
& +0.5 \exp \left(-\frac{(9 x-7)^{2}}{4}\right)+0.2 \exp \left(-(9 x-4)^{2}\right) .
\end{aligned}
$$

These functions were firstly proposed in [7] and result from adapting to the univariate case test functions generally used in the multivariate interpolation of large sets of scattered data [13]. We apply the approximating operators $\mathcal{L}_{B_{m}}, \mathcal{L}_{\mathcal{B}}$ and $S_{B_{m}}$ on each function $f_{i}$ with $c=r^{l}$. The operator $S_{B_{m}}$ is defined as follows

$$
\left(S_{B_{m}} f\right)(x)=\sum_{i=0}^{N} A_{\mu, i}(x) P_{m}\left[f ; x_{i}, x_{i+1}\right](x)
$$

with $A_{\mu, i}(x)=\frac{\left|x-x_{i}\right|^{-\mu}}{\sum_{k=0}^{N}\left|x-x_{k}\right|^{-\mu}}$, see [7] for details.

We use uniform grids of 17 points for $S_{B_{1}}, \mathcal{L}_{B_{1}}$, grids of 11 points for $S_{B_{2}}, \mathcal{L}_{B_{2}}$, and $\mathcal{L}_{\mathcal{B}}$, and finally grids of 8 points for $S_{B_{3}}$ and $\mathcal{L}_{B_{3}}$. In order to estimate the errors as accurate as possible, we compute the approximating functions at the points $\frac{i}{101}(i=1, \cdots, 100)$. Tables $1-6$ display mean and max errors for different values of the parameters $\mu, l$ and $m$. In these examples, we find that $c=r^{2}$ is an optimal parameter for the fixed precision. The numerical results show that the approximating power of the Bernoulli-type operator with multiquadrics is much better than that of the Shepard-Bernoulli operator. 


\section{Conclusions}

In this paper, a kind of Bernoulli-type quasi-interpolation operator is proposed by combining a univariate multiquadric quasi-interpolation operator with the generalized Taylor polynomial. A result on the convergence rate of the operator is given. Numerical experiments show that the operator is capable of producing high accuracy. Moreover, the associated algorithm is easily implemented.

In our future work, we plan to use the operator to fit scattered data. For instance, we are applying it to the fitting of discrete solutions of initial value problems of ODEs, and good results are obtained.

Acknowledgments. The work was partly supported by the National Natural Science Foundation of China (Grant Nos. 60533060, 10726068 and 10801024) and the Innovation Foundation of the Key Laboratory of High-Temperature Gasdynamics of Chinese Academy of Sciences, China.

\section{REFERENCES}

[1] R.K. Beatson and N. Dyn, Multiquadric B-splines, J. Approx. Theory, 87 (1996), 1-24.

[2] R.K. Beatson and M.J.D. Powell, Univariate interpolation on a regular grid by a multiquadric plus a linear polynomial, IMA J. Numer. Anal., 12 (1992), 107-133.

[3] R.K. Beatson and M.J.D. Powell, Univariate multiquadric approximation: quasi-interpolation to scattered data, Constr. Approx., 8 (1992), 275-188.

[4] M.D. Buhmann, Convergence of univariate quasi-interpolation using multiquadrics, IMA J. Numer. Anal., 8 (1988), 365-383.

[5] M.D. Buhmann, Multivariate interpolation in odd-dimensional Euclidean spaces using multiquadrics, Constr. Approx., 6 (1990), 21-34.

[6] M.D. Buhmann, Multivariate cardinal interpolation with radial-basis functions, Constr. Approx., 6 (1990), 225-255.

[7] R. Caira and F. Dell'Accio, Shepard-Bernoulli operators, Math. Comp., 76 (2007), 299-321.

[8] F. Costabile, Expansions of real functions in Bernoulli polynomials and applications, Conf. Semin. Math. Univ. Bari, 273 (1999), 1-13.

[9] R. Franke, Scattered data interpolation: tests of some methods, Math. Comp., 38 (1982), 181-200.

[10] R.L. Hardy, Multiquadric equations of topography and other irregular surfaces, J. Geophysical Res., 76 (1971), 1905-1915. 
[11] C.A. Micchelli, Interpolation of scattered data: distance matrices and conditionally positive definite function, Constr. Approx., 2 (1986), 11-22.

[12] M.J.D. Powell, Univariate multiquadric approximation: reproduction of linear polynomials, In: Multivariate Approximation and Interpolation (W. Haussman and K. Jetter, eds.), Basel: Birkhāuser Verlag, (1990), 227-240.

[13] R.J. Renka and A.K. Cline, A triangle-based $C^{1}$ interpolation method, Rocky Mountain J. Math., 14 (1984), 223-237.

[14] Z. Wu and R. Schaback, Shape preserving properties and convergence of univariate multiquadrics quasi-interpolation, Acta Math. Appl. Sinica (Engl. Ser.), 10 (1994), 441-446.

[15] W.X. Zhang and Z.M. Wu, Some shape-preserving quasi-interpolants to non-uniformly distributed data by MQ-B-splines, Appl. Math. J. Chinese Univ. Ser. B, 19 (2004), 191202. 